\title{
Solar Dynamical Processes II
}

\author{
Ashish Mishra $^{1 *}$, Mukul Kumar ${ }^{1,2^{*}}$ \\ ${ }^{1}$ Department of Physics, Indian Institute of Science Education and Research Bhopal, India. \\ ${ }^{2}$ State Key Laboratory of Space Weather, National Space Science Center, \\ Chinese Academy of Sciences, Beijing, China.
}
*Corresponding Author email:
${ }^{1}$ ashu.mishra274@gmail.com
2 mk.cas@yahoo.com

Article History

Received: 18 August 2018

Revised: 05 January 2019

Accepted: 03 February 2019

Published: 11 February 2019

Student(s)

- $\quad$ Ashish Mishra

Academic Year: 2015-16, 2 $2^{\text {nd }}$ Semester

Course Level: Master

Course Name: BS-MS dual degree program

Course year: $4^{\text {th }}$ Year $/ 8^{\text {th }}$ Semester

$\operatorname{Mentor}(s)$

- Mukul Kumar

\begin{abstract}
The present article is the successor of Solar Dynamical Processes I. The previous article was focused on the Sun, its magnetic field with an emphasis on various dynamical processes occurring on the Sun, e.g. sunspots, prominence and bright points which in turn plays a fundamental role in regulating the space weather. This article is emphasized on the solar dynamical processes and develop an extensive understanding of the various phenomena involved in their origin. The article also covers various models and hypothesis put forward by pioneer scientists on the basis of their observation by space-borne and groundbased instruments. This article shade light over a wide range of dynamical processes e.g., solar flares, coronal mass ejections, solar jets and coronal holes. Solar jets, the small-scale transient activities are found to have association with the other transient activities (e.g., mini-flares and mini-filaments). Flares as well as the coronal mass ejections are responsible for releasing a large amount of high energy charged particles and magnetic flux into the interplanetary space and are being considered as the main drivers of space weather.
\end{abstract}

Keywords: Solar Dynamical Processes, Solar Flare, Coronal Mass Ejection, Solar jets, Coronal Holes

\section{Introduction}

Solar dynamical processes are the primary drivers of space weather. They are the main source of any change in the solar atmosphere as well as in interplanetary space. The article Solar Dynamical Processes I [1] was focused on Sun and its properties in which we presented a brief overview of the solar magnetic field and developed an understanding of differential rotation in the Sun. The solar magnetic field is the governing factor for the dynamical processes taking place on the Sun. Unlike Earth's uniform poloidal magnetic field, the magnetic field of the Sun is non-uniform. The Sun possesses poloidal as well as toroidal magnetic field, which together provide a very non-uniform magnetic field distribution over photosphere/heliosphere [1] - [12]. According to the standard model, different layers of the Sun rotate at different angular velocity. This property of Sun is called differential rotation. Differential rotation and non-uniform magnetic field altogether are the two major factors 
responsible for dynamical processes such as sunspots, prominences, bright points, solar jets, coronal holes, solar flares, coronal mass ejections etc.

We also discussed dynamical processes like sunspots, prominences, bright points, giving a preliminary introduction to their properties and the factors responsible for their formation and stability. Sunspots are the cool, dark and magnetically constrained regions found on the surface of the Sun [13], [14]. They are normally found in the pairs/groups and have been reported to move from higher latitude to equator over the course of solar cycle [13]. Solar prominences are the arch-like structure having a large amount of plasma. They are often found high into the chromosphere and are supported by the magnetic tension [14]. They are cooler than the photosphere and are seen as dark as 'filament' like structure when observed against the disk [14]. Bright points are the short-term bright spots found mostly in the active region where magnetic reconnection can easily take place. They often indicate the presence of a magnetic field in the region and are associated with other dynamical processes such as solar jets, solar flares etc. [16]. In the present article, we will emphasize on other dynamical processes e.g., coronal holes, solar jets, solar flares and coronal mass ejections (CMEs).

\section{Coronal holes}

Coronal holes are large dynamic structures found at the polar caps and low latitude on the Sun (see figure 1). They are often identified as dark and cool structures having large scale open-magnetic field, very low density and temperature [17] - [19]. "Coronal holes are defined as dark regions on the space images of the Sun in UV spectral lines or in X-ray continuum images or (as bright regions) from the ground-based observations in the IR He I line $10830 \AA$ ” [20].

Observations and analysis of data suggested that coronal holes rotate like a rigid body [21], [22]. Based on a current-free model of the coronal magnetic field, in 1988, Nash et al. [23] suggested that magnetic reconnection can be helpful in understanding the rotation of coronal hole. In their model, they found that field-line reconnection can lead to quasi-rigid rotation of coronal holes and concluded that strong axisymmetric field components can support prolonged meridional coronal hole rotations during the period of decreasing solar activities. Wang and Sheeley [24] studied the interchange reconnection between open and closed magnetic flux at coronal hole boundaries. They suggested that the interchange reconnection oppose the shearing of magnetic flux and lead to quasirigid rotation of coronal holes.

Coronal holes largely depend on the solar cycle, i.e., during solar maxima, there are almost no polar coronal holes, while during solar minima they are much more prominent [25], [26]. An observational study of coronal holes during the solar cycle revealed that the temperature of coronal hole varies over the solar cycle with typical values of $1.17 \pm 0.06 \mathrm{MK}$ and $1.09 \pm 0.02$ MK during solar maximum and minimum, respectively [26]. This study also revealed the displacement of coronal holes shortly after its establishment at the poles, suggesting

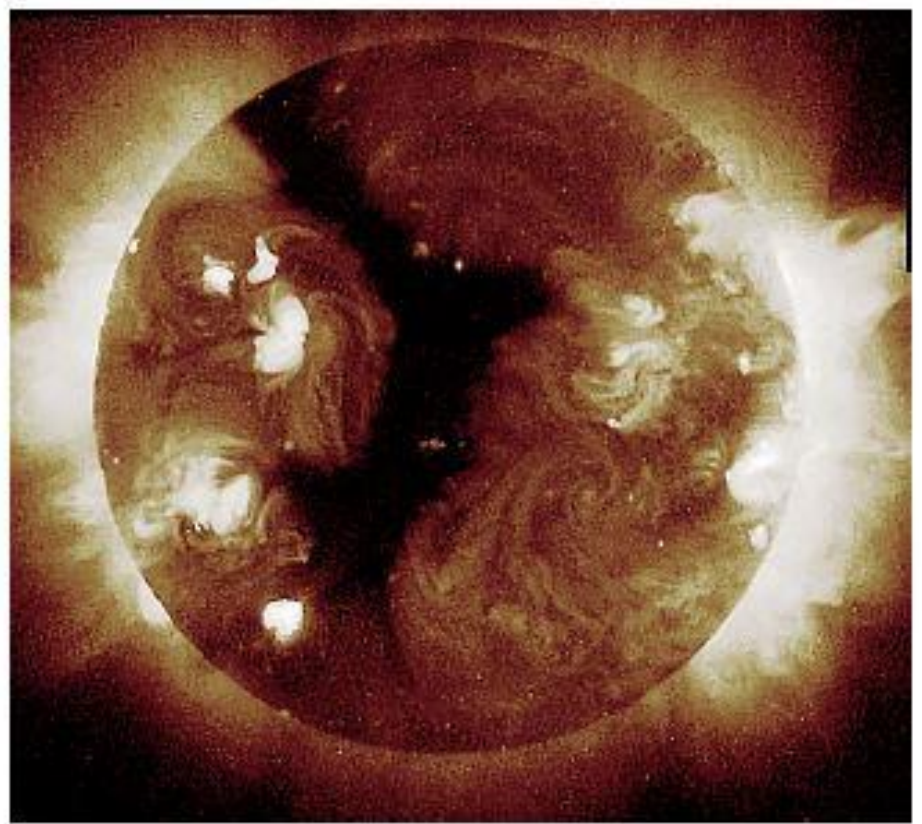

Figure 1: X-corona, image taken SXT-Yohkoh, Opted form Cranmer 2009 [17] 
Mishra et al., Adv. J. Grad. Res.; Vol. 6 Issue 1, pp: 1-13, July 2019

that the interaction between mid-latitude coronal holes of different polarities may lead to displacement. Based on the Skylab magnetogram analysis the magnetic field strength of low latitude coronal holes has been measured and found to vary between $7.2 \mathrm{G}$ to 0.46 G [27]. However, Harvey and Sheeley [28], based on the observation of low-latitude hole of average magnetic field strength of $15 \mathrm{G}$, suggested that upper average magnetic field strength should be double of that suggested by Bohlin and Sheeley [27].

There exist many models and hypotheses proposed for the formation of coronal holes. In coronal models, coronal holes are usually defined as "the footpoints of the magnetic field open into the heliosphere" [29], [30]. In 1974, W.M. Glencross [31] suggested that coronal holes are formed when two or more flux tubes of opposite polarities merge together to form a cavity in the magnetic field. The author also suggested that time scale of this process is approximately 24 hours. Later in 1975, Timothy et al., [22] suggested that coronal holes are formed by continuous emergence and dispersion of active region magnetic field. This process gives rise to large-scale unipolar magnetic field and occurs over a time scale of few solar rotations. They further suggested that the divergent configuration of coronal hole is obtained by the opposite polarity magnetic field surrounding the unipolar magnetic features. This hypothesis of Timothy et al. [22] was later confirmed by Bohlin and Sheeley [27], Harvey and Sheeley [28] and the citations therein.

Bohlin and Sheeley [27] gave the unbalanced flux model for equatorial holes and suggested that the coronal holes are formed when the unbalanced flux/ bipolar magnetic regions (BMR) come closer to form a balanced flux region. In their model, the application of unbalanced flux to other solar cycle phenomena like the movement of sunspots, polarity reversal in sunspots during new cycles, rapid activities during solar maxima etc., could predict the coronal holes throughout the solar cycle. A review by Harvey and Sheeley [28] suggested that the distribution of large-scale photospheric magnetic flux is the key factor in determining the structure and evolution of coronal holes. Their analysis of magnetic field revealed that a large number of fragmented finestructure elements organize to form a large-scale structure in the photosphere with a large area and dominated unipolar magnetic field over the period of one day. In 1996, Wang et al., [32] suggested that magnetic reconnection could take place near coronal boundaries. Kriesta et al. [33] also studied the interaction of open and closed magnetic field at the coronal hole boundaries and their effect on small-scale changes. They determined the velocity of coronal hole displacement to be $\leq 2 \mathrm{~km} / \mathrm{s}$ which was in compliance with the magnetic reconnection rates of the order $\leq 2 \times 10^{-3}$ at the coronal holes boundary.

While observing the coronal holes at various wavelengths e.g., microwave, optical and EUV, Gopalswamy et al., [25] found that radio brightness enhancement was greater in equatorial and polar coronal holes than the quiet Sun and these radio brightness enhancements were associated with the unipolar open magnetic field in the coronal holes. Further, they concluded that high-temperature region (of the order $10^{4} \mathrm{~K}$ ) in the upper chromosphere is the source of enhanced radio emissions. Norton et al., [34] studied the SOHO/EIT data of low and mid latitude coronal holes. They found that a symbiotic relationship between the coronal holes and the active region. They suggested the coronal hole emerges from the unipolar field surrounded by the bipolar field in the active region. Yang et al., [35] studied the formation data of an equatorial coronal hole obtained from GOES-12/SXI, SOHO/EIT and SOHO/MDI. They found that the coronal hole was formed gradually over the period of 81 hours followed by decrease in the magnetic flux, temperature, SXR and EUV brightness. They noticed that during this process these quantities were approximately unchanged in the quiet region of the Sun. They suggested that the decrease in the magnetic flux can be due to the magnetic reconnection between the open and closed magnetic field in the region which can be marked as the origin of the coronal hole. The coronal holes have been identified as a source of high-speed solar wind [36], [37]. These solar winds carry energetic particles into the interplanetary space and play a fundamental role in governing space weather.

Raju et al., [38] studied the physical conditions of coronal holes and the nearby regions by exploiting the SOHO/EIT and Norikura Solar Observatory data. They found that the nonthermal velocity in the coronal 
Solar Dynamical Processes II

holes was greater than that of the quiet region and suggested that the enhanced nonthermal velocity may have significant implications in the acceleration of high-speed solar winds. Subramanian et al., [39] studied the smallscale evolution of coronal hole boundaries and found that the coronal hole boundaries are dominated by a large number of brightening events. They suggested that magnetic reconnection between open and closed magnetic field lines are responsible for the outflow of plasma at larger distances. They further suggested that these outflows might act as one of the sources of the solar winds. In 2011, G. De Toma [29] analyzed the coronal holes during the solar minimum between the solar cycle 23 and 24 . The author found that most of the coronal holes present at low latitudes were a good source of high-speed solar wind streams. Karachik et al. [40] studied the correlation between the solar wind speed and coronal holes parameters and found a strong correlation between the solar wind speed and the area of coronal holes. They found that the number of coronal bright points and magnetic bipoles were gradually increased towards the center. They suggested that the contribution of near-equatorial parts of the coronal hole was very significant in driving high-speed solar wind. However, they further concluded that magnetic reconnection events, which are associated with coronal bright points, couldn't act as the main driver of the fast solar wind.

It is found that MHD waves play important role in coronal holes. Recently, Banerjee and Prasad [41] presented a short review of the observations of MHD waves in coronal holes. In the review, they stated that there are two main types of MHD waves, which dominate the coronal holes, compressive MHD waves and incompressive MHD waves. Compressive waves are the perturbations which further leads to density fluctuations while incompressive waves do not cause fluctuations in the intensity rather they cause fluctuations in other spectral data and can be identified through spectroscopic observations [41]. At the bottom of the coronal hole, Alfven waves can be formed in the small-scale magnetic field and give rise to the heating of coronal holes [42]. Coronal holes cannot be distinguished from the non-hole region very easily and temperature higher than $10^{5} \mathrm{~K}$ is required for the better distinguishable observations [17]. However, with better observational techniques, it is possible to identify coronal holes quantitively as well as qualitatively. It has been seen that the wavelength of observation is also important to observe and distinguish coronal holes from chromosphere [17], [20], [25]. Coronal holes have been observed to be one of the major sites of magnetic reconnection. This gives rise to other solar activities such as bright points, solar jets etc. [39], [40], [43], [44]. Gopalswamy et al., [45] studied the interaction of coronal mass ejection (CME) with coronal holes. Their study was based on the parameters such as area and average magnetic field of coronal holes and their distance from the origin of CMEs. They found that coronal holes could deflect the CMEs away from the normal Sun-Earth line. They also found that coronal hoes mostly influenced the CMEs with driverless shock while for the shocks with drivers, there was no significant effect of nearby coronal holes on CME. Their result was further supported by the findings of Mohamed et al., [46] and Makela et al. [47]. Wood et al., 2012 studied the STEREO data of shock driven by CMEs and found that they were deflected by the coronal holes. This result was consistent with the one reported by Gopalswamy et al., [45]. The study of CMEs and their forecast is very important, as they are one of the major drivers of geomagnetic storms on Earth. The correlation of coronal holes with CMEs and solar winds makes the study of coronal holes very important, as coronal holes are a source of the movement of charged matter and magnetic flux into the interplanetary space.

\section{Solar Jets}

Solar jets are very common in the heliosphere. They are characterized by the sudden high-speed collimated outflow of matter, magnetic flux and magnetic energy often accompanied with high temperature (up to the order of $10^{4}$ to $10^{6} \mathrm{~K}$ ). They are found all over the heliosphere and their physical properties vary according to their location/region on the Sun. There are many types of jet observed in the heliosphere depending upon their 
Mishra et al., Adv. J. Grad. Res.; Vol. 6 Issue 1, pp: 1-13, July 2019

location, properties and wavelength of observation like solar spicules, surges, polar jets (found in polar region), EUV jets (when observed in EUV wavelength), X-Ray jets (when observed using X-ray wavelength), etc.

Wang et al [48] studied the polar jets in detail using the white light data of LASCO/SOHO. They found most white light/EUV jets near the bright points indicating close relationship of jets with the interaction of magnetic field. They suggested that EUV jets were the manifestation of magnetic reconnection between the unipolar magnetic concentration and small bipolar magnetic field. They found that the number of jets exceeded 3-4 per day, having outward propagation speed ranging from 400 to $1100 \mathrm{~km} / \mathrm{s}$ with centroidal velocity ranging from 140 to $360 \mathrm{~km} / \mathrm{s}$. They also found that most of the jets erupt in the heliosphere with velocity less than that of escape velocity with an average outward speed of approximately $250 \mathrm{~km} / \mathrm{s}$. They further suggested that the study of jets could be helpful in characterizing the properties of polar winds. Shibata et al., [49] analyzed the Yohkoh-SXT data and noticed the existence of X-ray jets in the solar corona. They found that the coronal Xray jets were mostly associated with X-ray bright points, emerging flux and active regions and had physical parameters such as translational velocity, size and kinetic energy in the ranges $3-300 \mathrm{~km} / \mathrm{s}, 5 \times 10^{3}-4 \times 10^{5} \mathrm{~km}$ and $10^{25}-10^{28} \mathrm{erg}$ respectively. They suggested three possible origins of X-ray jets: jets by evaporation flow; magnetic twist; and reconnection with mixed hot and cold plasmas, in which required energy is mainly provided by the magnetic reconnection.

X-ray jets have been extensively studied using Yohkoh-SXT data [50] - [54]. Shimojo et al. [51] performed a statistical study of $100 \mathrm{X}$-ray jets images obtained by Yohkoh-SXT and found that most of the X-ray jets occur near active region and small flare sites. They estimated the length and width in the range $10^{4}-5 \times 10^{5} \mathrm{~km}$ and $5 \times 10^{3}-10^{5} \mathrm{~km}$ with apparent velocity in the range $10-1000 \mathrm{~km} / \mathrm{s}$ (average $200 \mathrm{~km} / \mathrm{s}$ ). They also found the occurrence rate of X-ray jets to be approximately 20 per month with a lifetime in the range $100 \mathrm{~s}$ to $16000 \mathrm{~s}$. Studied have reported that the density and temperature vary in the range $0.7-4 \times 10^{9} \mathrm{~cm}^{-3}$ (average: $1 \times 10^{9} \mathrm{~cm}^{-}$ ${ }^{3}$ ) and $3-8 \mathrm{MK}$ (average: 5.6 MK) respectively with thermal energy in the range 1027 - $10^{29} \mathrm{ergs}$ [52] - [54]. Savcheva et al, [55] analyzed the Hinode/XRT data of the polar jets within the polar coronal holes. They found that the frequency of solar X-ray jet occurrence to be on average 60 per day with the majority of jets occurring inside the coronal hole. They carried out a statistical study of the physical properties of the polar jets such as height, collimated width, velocity and lifetime and found average values of $50000 \mathrm{~km}, 8000 \mathrm{~km}, 160 \mathrm{~km} / \mathrm{s}$ and 10 min, respectively. They also investigated the transverse velocity of the jets and defined it as "the velocity of the translation motion of the jet in the direction perpendicular to its elongation" and measured the transverse velocity in the range from 0 to $35 \mathrm{~km} / \mathrm{s}$. An analysis of SECCHI/EUV data of EUV coronal jets near-equatorial region by Nistico et al., [56], [57] revealed that the lifetime of EUV jet is typically between 20-40 min with velocity in the range 270 to $400 \mathrm{~km} / \mathrm{s}$. These observations have been summarized in Table-1.

Table 1: Jet properties as observed in different instruments. Numbers in parenthesis are the average values. Opted from Torok et al. [58], Parachiv et al. [59], Savcheva et al., [55]

\begin{tabular}{|c|c|c|c|}
\hline Jet Property & $\mathbf{S X T}^{\text {[51], [52], [54] }}$ & $\mathbf{X R T}^{[55],[59],[60]}$ & EUVI $^{[56],[57]}$ \\
\hline Source Region & Active Region & Polar coronal hole & Polar/Equatorial coronal hole \\
\hline Occurrence & -20/month & $\sim 60 /$ day & $-\ldots$ \\
\hline Duration (min) & $2-600$ & (10) & $20-40$ \\
\hline Velocity $(\mathrm{km} / \mathrm{s})$ & $10-1000(200)$ & $70-400(160)$ & $270-400$ \\
\hline Length $(\mathrm{Mm})$ & $30-400(150)$ & $10-120(50)$ & 100 \\
\hline Width (Mm) & $5-100(70)$ & $6-10(8)$ & 25 \\
\hline Density $\left(10^{8} \mathrm{~cm}^{-3}\right)$ & $7-40(17)$ & $1.5 \pm 0.1$ & $-\cdots$ \\
\hline Temperature (MK) & $3-8(5.6)$ & $1.8 \pm 0.2$ & - - - \\
\hline
\end{tabular}


Cirtain et al., [60] exploited the Hinode/XRT data of polar coronal holes for analyzing the X-ray jets in the nearby regions. They reported that on an average $10 \mathrm{X}$-ray jet events were observed per hour having two velocity components of the order of Alfven speed $(\sim 800 \mathrm{~km} / \mathrm{s})$ and sound speed $(\sim 200 \mathrm{~km} / \mathrm{s})$, respectively. However, their lifetime was found to be in range from 100 to $1500 \mathrm{~s}$. They suggested that the high velocity of $\mathrm{X}$-ray jets was achieved by the magnetic reconnection and these sites can be a source of Alfven waves.

It is believed that coronal jets are formed by the interaction of the magnetic field between open (unipolar) and closed (bipolar) magnetic fields in the solar corona [58], [61]. Yokoyama and Shibata [62]-[64] carried out a two-dimensional MHD simulation of magnetic reconnection between emerging magnetic flux and the overlying coronal field. They suggested that different coronal magnetic field orientation (horizontal or oblique) give rise to different type of jets (horizontal or oblique) (see figure 2). In the study, they found that the cool plasma is carried upward by the emerging flux and accelerated by magnetic tension that give rise to cool jets. Emphasizing the importance of location of reconnection, they further suggested that nearer the reconnection to the photosphere, more the energy is released giving rise to hotter jet. However, if the reconnection takes place in the chromosphere, then it is more likely to produce cool jet. They further proposed the association of solar jets with small or micro-flares. Interaction of emerging flux and pre-existing magnetic flux has been widely studied [65]- [68].

(b)

(a)

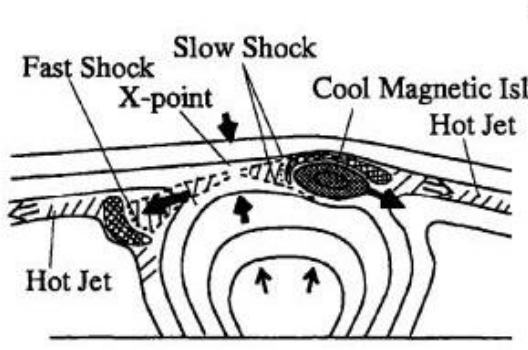

(c)

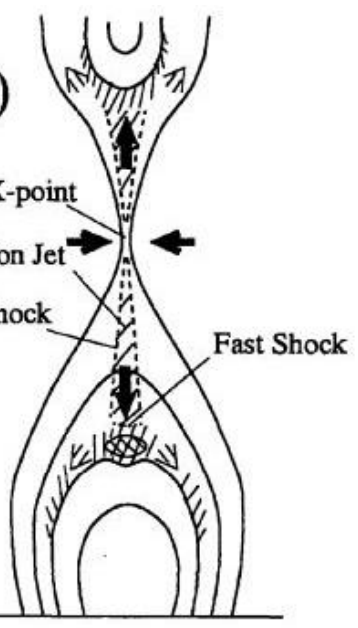

Figure 2: Schematic topology of (a) simulation with horizontal jets, (b) simulation with oblique jet, and (c) flare model, Opted from Yokoyama and Shibata 1996, [63]

Gontikakis et al., [69], [70] performed threedimensional MHD simulation of flux emergence and its reconnection with preexisting magnetic flux. Figure 3 shows the magnetic geometry of the simulation in which the preexisting magnetic field in the active region (marked as 1 in figure 3) undergoes

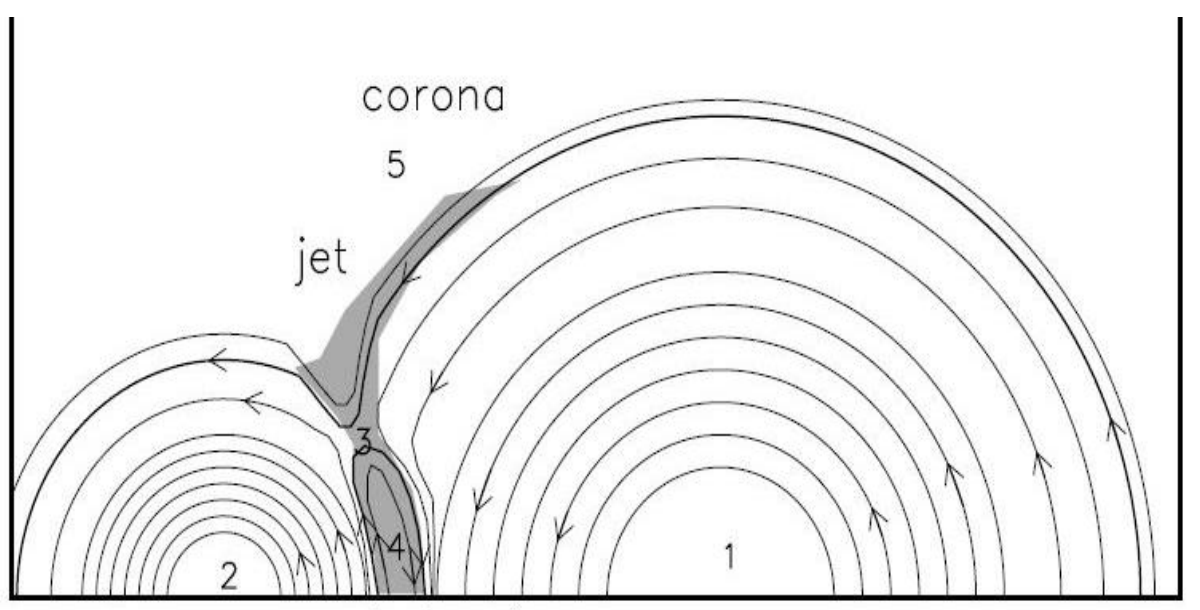

Figure 3: Magnetic geometry of the simulation, opted form Gontikikas et al. 2010, [70] 
Mishra et al., Adv. J. Grad. Res.; Vol. 6 Issue 1, pp: 1-13, July 2019

reconnection with the emerging field (marked as 2 in figure 3). Following the reconnection processes, reconnection current sheet is formed (marked as 3 in figure 3) followed by small reconnection loop (marked as 4 in figure 3) and upper domain of jet (marked as 5 in figure 3). They found that the reconnection site give rise to high velocity upflow (jet) which followed the active region loop and subsequently falling into the solar surface, (see figure 3: jet plasma follows the magnetic field lines in above pre-existing field (above region 1 in domain 5) and subsequently falls in the solar surface). They compared it with the observations of active region jet obtained from TRACE, SUMER and MDI magnetogram and concluded that the jets are produced by reconnection processes on the appearance of emerging flux.

Pariat et al., [71] proposed a model for generation of polar coronal jets by performing the three-dimensional simulation in which axis-symmetric null point topology cannot undergo magnetic reconnection. They found that the kink instability could cause magnetic reconnection between twisted closed and untwisted open magnetic field leading to impulsive release in energy, which can help in the generation of solar jets.

Moore et al., [72] analyzed the Hinode/XRT coronal jets and found different kind of "blowout jets" than the "standard X-ray jets". They suggested that blowout jets are formed by the ejective eruption of the base arch when the adequately large twisted magnetic flux tubes erupt to form jet due to reconnection and eruption. Pillet et al. [73] exploited the Hinode Spectropolarimeter data and analyzed quiet Sun jets. They found that jets were often located on the outer boundary of the granules and concluded that jets are formed by the interaction of horizontal field lines with the emerging field at the granular scale.

Recently, Pariat et al. [74] examined the impact of plasma $\beta$ on the solar jet by performing three - dimensional MHD model. They found that plasma $\beta$ plays an important role in determining the morphology of the jet. They suggested that reconnection process in twisted magnetic field opens up the magnetic field lines. These open field lines release the twist present at the footpoints by the generation of propagating torsional Alfven waves which heat, compresses and accelerates the plasma during their propagation and thus giving rise to helical jet structures. Solar jets have been observed to be associated with many other dynamical processes [75], 76]. Opher et al., [77] studied the MHD instabilities and magnetic reconnection in the solar jets by performing MHD simulations and demonstrated that jets in the heliosphere are turbulent and can interact with the solar wind. Recent studies have reported that filament eruption at a smaller scale can also give rise to a jet [78], [79]. Recently, Wyper et. al. [79] studied the three - dimensional MDH simulation for mini - filament eruptions using the magnetic breakout model given by Antiochos et al., [80]. In their model, magnetic reconnection releases energy stored in filament channel which eventually erupts to form a blowout jet.

\section{Coronal Mass Ejection}

Coronal mass ejections (CMEs) are one of the most important solar dynamical processes, which vastly affect the space weather and were discovered in 1971 [81]. During a CME, a large amount of magnetic flux $\left(-10^{23}\right.$ $\mathrm{Mx})$ and mass $\left(-10^{16} \mathrm{~g}\right)$ is released into the interplanetary space with total energy exceeding $10^{32}$ ergs [82] - [86]. This rapid release is often identified by 3-part structure of CME, see Figure 4: (i) outer bright limb; (ii) cavity and (iii) the bright core [87].

Many models have been proposed on the origin of CMEs and their impact on the dynamics of the Sun and interplanetary phenomena. Van Tend and Kuperus [89]

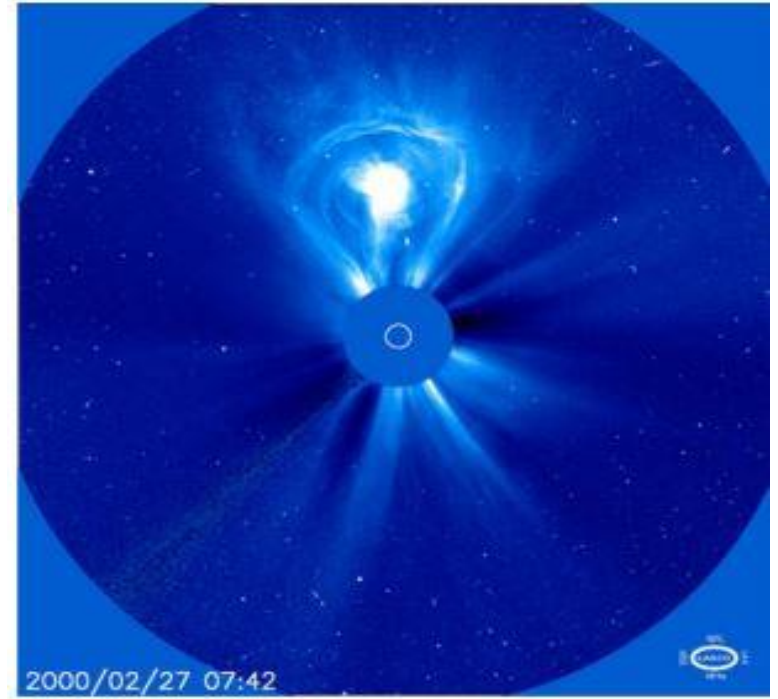

Figure 4: CME with its 3-parts structure, opted from Kilpua et al [88] 
proposed a catastrophe mechanism in which the loss of equilibrium due to exceeding current may trigger a CME. Forbes and Isenberg et. al., [90] studied the catastrophe mechanism given by VanTend and Kuperus [89] and suggested that the equilibrium is lost due to magnetic reconnection which increases the current and hence creating imbalance between magnetic tension and compression. This leads to the eruption of the filament that can further lead to a CME. Antiochos et al. [80] proposed a magnetic breakout model for the onset of coronal mass ejections. They performed a 2.5-dimensional simulation of their model consisting of multipolar topologies where the CME onsets. In their model, reconnection occurs in the shear arcade and neighbouring flux systems, which disrupts the equilibrium and triggers the CME. Karpen et al. [91] extended the magnetic breakout model [80] to perform a 2.5-dimensional simulation of breakout CME with high spatial resolution and concluded that the fast breakout reconnection onset CME while the fast acceleration of CME further onset the fast flare reconnection.

CME travels away from the Sun at very high speed and their speed depends on many factors e.g., the location of occurrence, energy, coronal drag etc. CME triggered towards the interplanetary system is called interplanetary CME or ICME. ICMEs faces aerodynamic drag from solar wind, and based on their speed, they face acceleration/deceleration from the solar wind [92] - [94]. CMEs are closely related to other dynamical processes on Sun such as solar jets, solar flares and prominence. It has been reported that the eruption of prominence often leads to a CME [95]. Liu et al. [96] observed and analyzed the data obtained from SDO, SOHO and STEREO and suggested that solar jet event can trigger a high - speed CME. Their observation was consistent with the three - dimensional model of solar jets proposed by Pariat et al. [71]. Makela et al. [97] studied the relationship between solar energetic particles and highly energetic CMEs produce heavy - ion event and have higher velocities. Gopalswamy et al., [85] suggested that the high energy CMEs are associated with reservoirs of a large amount of magnetic energy like sunspots.

In a much similar way as for other dynamical processes, magnetic reconnection plays a very important role in CME eruptions. Magnetic energy released is large enough to empower CMEs with large kinetic energy, with which it affects interplanetary space and space weather. CMEs may possess very high velocities, which can further accelerate or decelerate the solar wind. They also enrich solar winds with high-energy particles, which interact with Earth's magnetic field and gives rise to phenomenon like Aurora. When a large amount of energy is released during CME, they can give rise to geomagnetic storms. These geomagnetic storms can disrupt both grounds based as well as spaceborne instruments.

\section{Solar Flare}

Solar flares, the energetic events, are often identified by intense brightness due to a sudden burst of magnetic flux and solar mass into the solar corona (see figure 5). It is one of the most widely read solar phenomena, with its studies going back to the Carrington event in 1859 . Solar flares can occur anywhere on the Sun and have been associated to other dynamical processes such as CME, prominence eruption and bright points, for e.g., it has been reported in many studies that CME eruption can trigger a flare [98] - [101]

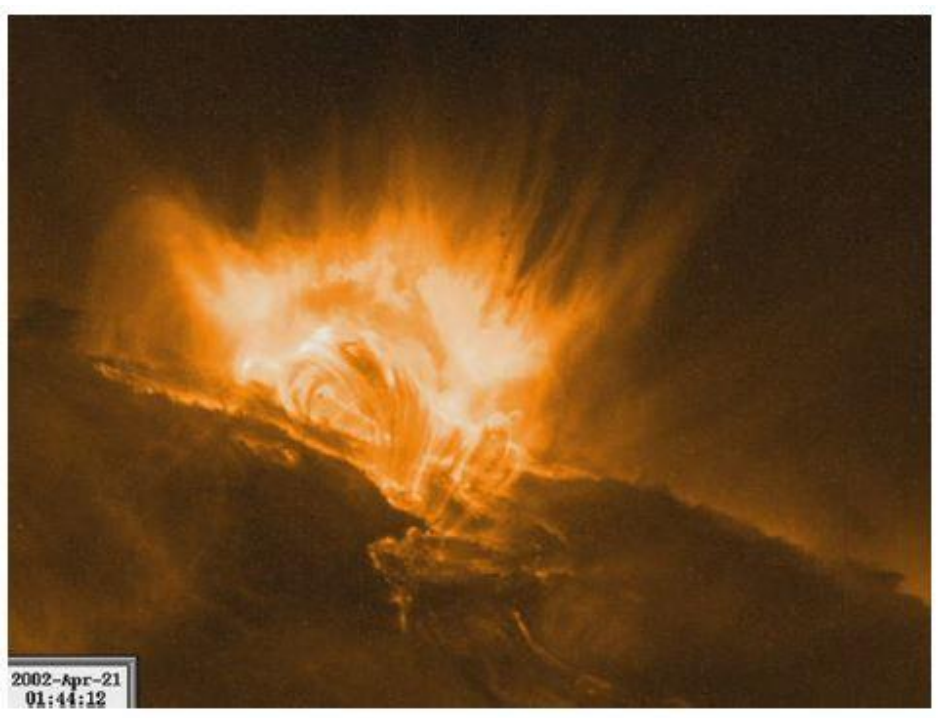

Figure 5: Observed solar flare on April 21, 2002. Opted from Benz [98] 
Mishra et al., Adv. J. Grad. Res.; Vol. 6 Issue 1, pp: 1-13, July 2019

It is now well established that magnetic reconnection or emergence can onset a flare. Researchers have suggested various models on the triggering mechanism of solar flares. Notably, the CSHKP model [102] - [105] consists of a large loop with fixed foot points, and the expansion of the magnetic loop triggers magnetic reconnection, which can erupt the loop giving rise to flare. Recently, Seaton et. al. [106] observed the current sheet structure of solar flare and the associated CME observations from SDO/AIA. In the CSHKP model, the formation of the current sheet is important for magnetic reconnection and eruption of flare. It is well established that CMEs and flares are very closely related to each other [98] - [101]. Gopalswamy et. al. [107], studied large flares which were not associated with CMEs and found intense microwave burst associated with the flares and suggested the production of non - thermal electrons.

Heyvaerts et al., [108] proposed an emerging flux model in which a flare event was a result of a continuous process divided into three phases: pre-fare heating, impulsive phase and main phase (see Figure 6). In the preflare phase, reconnection between the emerging flux and overlying magnetic field results in the formation of current sheets. Plasma starts heating up in the formed current sheet and rising emerging flux starts having plasma microinstabilities. In the impulsive phase, the acceleration of particles takes place along the magnetic field lines and thus reaching a critical current density in the current sheet which onsets a flare followed by the steady reconnection in the main phase.

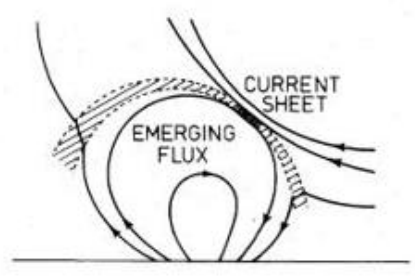

(a) Preflare Heating

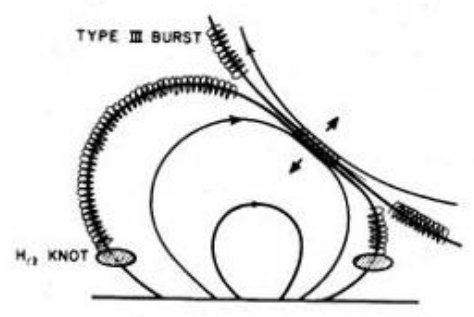

(b) Impulsive Phase

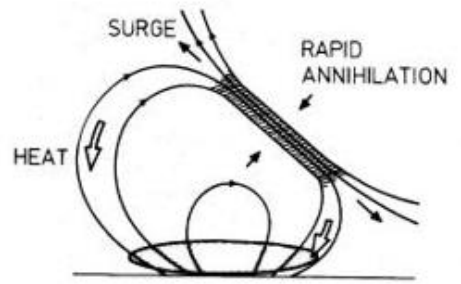

(c) Main Phase

Figure 6: Schematic diagram of (a) preflare phase, (b) Impulsive phase, and (c) Main phase, opted from Heyvearts et. al 1977, [108]

During a flare large amount of matter is released into the interplanetary space and emanate waves varying over the spectral range [109], [110] (see Table 2).

Table 2: Sources of waves in impulsive flares, Opted from Mishra and Kumar 2017 [82] and citations therein.

\begin{tabular}{|l|l|}
\hline Waves Produced & Sources \\
\hline Microwaves & Gyrosynchrotron emission from spiralling of electron \\
\hline Hard X-Ray and Gamma Ray & Bremsstrahlung from sub-relativistic and relativistic electron \\
\hline Radio wave low frequency & Plasma radiation \\
\hline Soft X-Ray & Thermal bremsstrahlung \\
\hline $\begin{array}{l}\text { Visible and Extreme Ultraviolet } \\
\text { Radiation }\end{array}$ & Hot thermal plasma \\
\hline Gamma Ray lines & Interaction of MeV ions with ambient nuclei \\
\hline Gamma Ray continuum & Decay of pions \\
\hline
\end{tabular}


Solar Dynamical Processes II

Solar flares are associated with many other dynamical processes. As already discussed, almost all CMEs are followed by soft X-ray jets [107]. Prominence eruption also triggers flares and CMEs. Study of X-ray jets has revealed the association of flares with microflares and plays important role in triggering a solar flare.

Magnetic reconnection provided most of the energy required for the eruption of flare. The magnetic energy released have very significant consequences as a large amount of magnetic flux and plasma is released into the interplanetary space. The energy released greatly contributes to the acceleration of solar energetic particles and solar winds which in turn affect space weather and may give rise to geomagnetic storms.

\section{Conclusion}

In this article, we discussed the dynamical processes with an emphasis on coronal holes, solar jets, solar flares, coronal mass ejections (CMEs) and their physical properties. We also discussed various models and their association with other dynamical processes. The article shed light on how the reconnection between closed and open field may lead to the formation of coronal holes. These holes have large open magnetic field spreading high into the heliosphere and have been reported to have the rotation. Magnetic reconnection at the boundary of coronal holes serves as the site of many other dynamical processes such as bright points and solar jets. Coronal holes have a close association with the solar wind. Advances in technology, observational techniques and instruments have enhanced our understanding of dynamical processes like solar jets, flares and CMEs. Solar jets are the long - collimated ejection of matter and have been reported to have the temperature of the order of $10^{6} \mathrm{~K}$. The article also encounters the role of magnetic reconnection and emerging magnetic flux in the formation of solar jets and its relationship with micro-flares. Mostly, Coronal Mass Ejections (CMEs) and flares are found to be very closely associated phenomena. It is very well established that flares often follow CMEs. In this article, we emphasized the relationship between the CMEs and flares with other dynamical processes. CMEs and flares follow most prominence eruption. Recently, it has been reported that jets can also trigger a CME.

\section{How to Cite this Article:}

A. Mishra and M. Kumar, “Solar Dynamical Processes II”, Adv. J. Grad. Res., vol. 6, no. 1, pp. 1-13, Feb. 2019. Doi: 10.21467/ajgr.6.1.1-13

\section{References}

[1]. A. Mishra and M. Kumar, "Solar Dynamical Processes I", Adv. J. Grad. Res., vol. 3, no. 1, pp. 47-61, Jan. 2018.

[2]. M. Schussler, "The Sun and Its Restless Magnetic Field", Lect. Notes Phys. 656, 23-49, 2005.

[3]. H. W. Babcock, H.D. Babcock, "The Sun's magnetic field, 1952 -1954”, The Astrophysical Journal, 121, $349,1955$.

[4]. H. W. Babcock, "The topology of the Sun's magnetic field and the 22-year cycle", The Astrophysical Journal, 133, $572,1961$.

[5]. H. W. Babcock, T.G. Cowling, "General magnetic fields in the Sun and stars", Mon. Not. R. Astr. Soc., 113, $357,1953$.

[6]. J. W. Dungey, "The motion of magnetic fields", MNRAS, 113, 1953.

[7]. R. B. Leighton, 1964, "Transport of magnetic fields on the Sun”, The Astrophysical Journal, 140, 1547, 1964.

[8]. R. B. Leighton, 1969, "A magneto-kinematic model of the solar cycle", The Astrophysical Journal, 156, 1969.

[9]. T. Wiegelmann, T. Sakurai, "Solar force free magnetic field”, arXiv: 1208.4693v1 [astro-ph.SR], 2012.

[10]. H. Zirin, "Fine Structure of Solar Magnetic Field", Solar Physics, 22, 34-48, 1972.

[11]. B. Tiwari and M. Kumar, “An Insight into the Solar Dynamo Theory”, International Annals of Science, vol. 3, no. 1, pp. $27-36$, Dec. 2017.

[12]. E. N. Parker, "Hydromagnetic Dynamo Models", The Astrophysical Journal, 122, 293, 1955.

[13]. S. K. Solanki, "Sunspots: A Review”, The Astronomy and Astrophysics Review, 11, 153-286, 2003.

[14]. J. M. Borrero, K. Ichimoto, "Magnetic structure of sunspots", Living reviews Solar physics, 8, 2011.

[15]. S. Parenti, "Solar prominence: Observations", Living Reviews in Solar Physics, 11, 1, 2014.

[16]. S. Pucci, G. Poletto, A.C. Sterling, M. Romoli, "Solar Polar X-ray jets and multiple bright points: Evidence for sympathetic activity", The Astrophysical Journal Letters, 745, L31 (5pp), 2012.

[17]. S. R. Cranmer, "Coronal Holes”, Living Reviews in Solar physics”, 6, 3. 2009.

[18]. J. B. Zirker, "Coronal Holes - An Overview", chhs.conf., 1977.

[19]. J. D. Bohlin, “An observational definition of coronal holes", chhs.conf., 1977.

[20]. V. G. Nagnibeda, "Coronal holes observed at short millimetre wavelength", ESASP, 415, 353N, 1997.

[21]. W. J. Wagner, "The rigid rotation of coronal holes", BAAS, 7,457, 1975.

[22]. A. F. Timothy, A.S. Krieger, G.S. Vaiana, "The structure and evolution of coronal holes", Solar Physics, 42, 135-156, 1975. 
Mishra et al., Adv. J. Grad. Res.; Vol. 6 Issue 1, pp: 1-13, July 2019

[23]. A. G. Nash, N. R. Sheeley Jr., Y.-M. Wang, "Mechanism for the rigid rotation of coronal holes", Solar Physics, 119, 359- $389,1998$.

[24]. Y. -M. Wang, N. R. Sheeley Jr., "Footpoint switching and the evolution of coronal holes", The Astrophysical Journal, Vol. 62, No. 2, 2004.

[25]. N. Gopalswamy, K. Shibasaki, C.B. DeForest, B.J.I. Bromage, G. Del Zanna, "Multiwavelength observation of a coronal hole", Synoptic Solar Physics, ASP Conference Series, Vol. 140, 1998.

[26]. S. A. Chapman, "The variation of coronal holes with the solar cycle", Obs, 128, 435C, 2008.

[27]. J. D. Bohlin, N. R. Sheeley Jr., "Extreme Ultraviolet observations of coronal holes. II - Association of holes with magnetic fields and a model for their formation during the solar cycle", Solar Physics, 56, 125 - 151, 1978.

[28]. J. H. Harvey, N. R. Sheeley Jr., "Coronal holes and solar magnetic fields, Space Science Review, 23, 139 - 158, 1979.

[29]. G. de Toma, "Evolution of coronal holes and implications for high-speed solar wind during the minimum between cycles 23 and 24", Solar Phys, 274, $195-217,2011$.

[30]. G Ge Toma, C. N. Arge, P. Riley, “Observed and modeled coronal holes,” ESA SP, 592, 2005.

[31]. G. W. Glencros, "Formation of holes in the solar corona", Nature, Vol. 250, 1974.

[32]. Y.M. Wang, S.H. Hawley, N.R. Sheeley Jr, "The magnetic nature of coronal holes", vol. 571, no. 5248, 464-469, 1996.

[33]. L. D. Krista, P. T. Gallagher, D. Shaun Bloomfield, "Short-term evolution of coronal hole boundaries", The Astrophysical Journal Letters, 731: L26 (5pp), 2011.

[34]. H. Norton, J. S. Newmark, U. Fieldman, "SOHO EIT observations of coronal holes", ESA SP, 446, 1999.

[35]. L. Yang, Y. Jiang, J. Zhang, "The formation of an equatorial coronal hole", Proceedings IAU Symposium, No. $264,2009$.

[36]. A.S. Krieger, A.F. Timothy, E.C. Roelof, "A coronal hole and its identification as the source of a high velocity solar wind stream", Solar Physics, 29, 505-525, 1973.

[37]. J.T. Nolte, A.S. Krieger, A.F. Timothy, R.E. Gold, E.C. Roelof, G. Vaiana, A.J. Lazarus, J.D. Sullivan, P.S. McIntosh, "Coronal holes as sources of solar wind", Solar Physics, 46, 303-322, 1976.

[38]. K. P. Raju, T. Sakurai, K. Ichimoto, J. Singh, "The physical conditions in a polar coronal hole and nearby regions from Norikura and SOHO observations", The Astrophysical Journal, 543, 1044 - 1050, 2000.

[39]. S. Subramanian, M. S. Madjarska, J. G. Doyle, "Coronal hole boundaries evolution at small scales II. XRT view. Can small scale outflows at CHBs be a source of the slow solar wind", Astronomy and Astrophysics, 516, 2010.

[40]. N. V. Karachik, A. A. Pevtsov, "Solar wind and coronal bright points inside coronal holes", The Astrophysical Journal, 735 : 45 (6pp), 2011.

[41]. D. Banerjee, S. K. Prasad, "MHD waves in coronal holes", arXiv: 1505.04475v1, 2015.

[42]. $\quad$ E.N. Parker, "Heating of coronal holes", The Astrophysical Journal, 372, 719-727, 1991.

[43]. Z. Huang, M. Madjarska, G. Doyle, D. Lamb, "Evolution of magnetic field corresponding to X-ray brightening events in coronal holes and quiet Sun", Proceedings IAU Symposium, No. 294, 2012.

[44]. G. A. Doschek, E. Landi, H. P. Warren, L. K. Harra, "Bright points and jets in polar coronal holes observed by the extreme-ultraviolet imaging spectrometer on Hinode", The Astrophysical Journal, 710: 1806 - 1824, 2010.

[45]. N. Gopalswamy, P. Makela, H. Xie, S. Akiyama, S. Yashiro, "CME interactions with coronal holes and their interplanetary consequences", Journal of Geophysical Research, Vol. 114, A00A22.

[46]. A. A. Mohamed, N. Gopalswamy, S. Yashiro, S. Akiyama, P. Makela, H. Xie, H. Jung, "The relation between coronal holes and CMEs during the rise, maximum, and declining phases of solar cycle 23", Journal of Geophysical Research, Vol. 117, A01103, 2012.

[47]. P. Makela, N. Gopalswamy, H. Xie, A. A. Mohamed, S. Akiyama, S. Yashiro, "Coronal holes influence on the observed structure of interplanetary CMEs", arXiv: 1301.2176v1, 2013.

[48]. Y. -M Wang, N. R. Sheeley Jr., D.G. Socker, R. A. Howard, G. E. Brueckner, D. J. Michels, D. Moses, O. C. St. Cyr, A. Llebaria, J. P. Delabouldiniere, "Observations of Correlated White-Light and Extreme-Ultraviolet Jets from Polar Coronal Holes", The Astrophysical Journal, Volume 508, Issue 2, pp. 899-907, 1998.

[49]. K. Shibata, Y. Ishido, L. W. Acton, K. T. Strong, T. Hirayama, Y. Uchida, A. H. McAllister, R. Matsumoto, S. Tsuneta, T. Shimuzu, H. Hara, T. Sakurai, K. Ichimoto, Y. Nishino, Y. Ogawara, "Observations of X-ray jets with the Yohkoh Soft X-ray telescope”, PASJ, 44, L173-L179, 1992.

[50]. K. Shibata, M. Shimojo, T. Yokoyama, M. Ohyama, "Theory and observations of X-ray jets", APS Conference Series, Vol. $111,1996$.

[51]. M. Shimojo, H. Hashimoto, K. Shibata, T. Hirayama, H. S. Hudson, L. W. Acton, "Statistical Study of Solar X-Ray Jets Observed with the YOHKOH Soft X-Ray Telescope", Publications of the Astronomical Society of Japan, v.48, p.123-136, 1996.

[52]. M. Shimojo, K. Shibata, K. Hori, T. Yokoyama, "Physical parameters of solar X-ray jets", ESA SP, 421, 1998.

[53]. K. Shibata, "Theory of flares and MHD jets", Proceedings of IAU Symposium, 188, 1998.

[54]. M. Shimojo, K. Shibata, "Physical parameters of solar X-ray jets", The Astrophysical Journal, 542, 1100 - 1108, 2000.

[55]. A. Savcheva, J. Cirtain, E. E. DeLuca, L. L. Lundquist, L. Golub, M. Weber, M. Shimojo, K. Shibasaka, T. Sakao, N. Narukage, S. Tsuneta, R. Kano, "A Study of Polar Jet Parameters Based on Hinode XRT Observations", PASJ, 59, S771 - S778, 2007.

[56]. G. Nistico, V. Bothmer, S. Patsourakos, G. Zimbardo, "Characteristics of EUV coronal jets observed with STEREO/SECCHI", Solar Physics, 259:87, 2009.

[57]. G. Nistico, V. Bothmer, G. Zimbardo, S. Patsourakos, "Observational features of equatorial coronal hole jets", Annales Geophysicae, Volume 28, Issue 3, 2010, pp.687-696, 2010.

[58]. T. Torok, R. Lionello, V. S. Titov, J. E. Leake, Z. Mikic, J. A. Linker, M. G. Linton, "Modeling jets in the corona and solar wind", arXiv:1511.09350v1, 2015. 
Solar Dynamical Processes II

[59]. A. R. Parachiv, A. Bemporad, A. C. Sterling, "Physical properties of solar polar jets: A statistical study with Hnode XRT data", arXiv:1505.07191v1, 2015.

[60]. J. Cirtain, L. Golub, L. Lundquist, A. van Ballegooijen, A. Savcheva, M. Shimojo, E. DeLuca, S. Tsuneta, K. Reeves, M. Weber, R. Kano, N. Narukage, K. Shibasaka, "Evidence of Alfven waves in solar x-ray jets", Science, Volume 318, Issue 5856, pp. 1580, 2007.

[61]. D. Dobrzycka, "Solar Coronal Jets", Mem S. A. It., Vol. 78, 268, 2007.

[62]. T. Yokoyama, K. Shibata, "Magnetic reconnection as the origin of X-ray jets and Ha surges on the Sun", Nature, Volume 375, Issue 6526, pp. 42-44, 1995.

[63]. T. Yokoyama, K. Shibata, "Numerical Simulation of Solar Coronal X-Ray Jets Based on the Magnetic Reconnection Model", Publications of the Astronomical Society of Japan, v.48, p.353-376, 1996.

[64]. T. Yokoyama, K. Shibata, "MHD Simulation of Solar Coronal X-ray Jets: Emerging Flux Reconnection Model", Astrophysical Letters and Communications, Vol. 34, p.133, 1996.

[65]. T. Miyagoshi, T. Yokoyama, H. Isobe, K. Shibata, "Jet Phenomena in the Solar Atmosphere caused by Interaction between Emerging Flux and Coronal Fields", ASP Conference Series, Vol. 325, 2004.

[66]. K. Galsgaard, F. Moreno - Insertis, V. Archontis, A. Hood, “A Three-dimensional Study of Reconnection, Current Sheets, and Jets Resulting from Magnetic Flux Emergence in the Sun”, The Astrophysical Journal, Volume 618, Issue 2, pp. L153-L156, 2005.

[67]. F. Moreno - Insertis, "X-ray jets and magnetic flux emergence in the Sun", Proceedings IAU Symposium No. $259,2008$.

[68]. J. Y. Ding, M. S. Madjarska, J. G. Doyle, Q. M. Lu, K. Vanninathan, Z. Huang, "Magnetic reconnection resulting from flux emergence: Implications for jet formation in the lower solar atmosphere?", arXiv: 1109.5606v1, 2011.

[69]. C. Gontikakis, V. Archontis, K. Tsinganos, "Observations and 3D MHD simulations of a solar active region jet", A\&A, 506, 45 - 48, 2009.

[70]. C. Gontikakis, V. Archontis, K. Tsinganos, "Study of a solar active region jet, ASP Conference Series, Vol. 424, 2010.

[71]. E. Pariat, S.K. Antiochos, C.R. DeVore, "A model for solar polar jets", The Astrophysical Journal, 691, 61-74, 2009.

[72]. R. L. Moore, J. W. Cirtain, A. C. Sterling, D. A. Falconer, "Dichotomy of Solar Coronal Jets: Standard Jets and Blowout Jets", The Astrophysical Journal, Volume 720, Issue 1, pp. 757-770, 2010.

[73]. V. M. Pillet, J. C. del Toro Iniesta, C. Q. Noda, "Ubiquitous quiet Sun jets", arXiv:1104.5564.v1, 2011.

[74]. E. Pariat, K. Damasse, C. R. DeVore, S. K. Antiochos, J. T. Karpen, “A model for straight and helical solar jets II. Parametric study of the plasma beta", Astronomy \& Astrophysics, Volume 596, id.A36, 20 pp, 2016.

[75]. N. M. Hoekzema, R. J. Rutten, J. W. Cook, "Ultraviolet Jets and Bright Points in the Solar Chromosphere. II. Statistical Correlations", The Astrophysical Journal, Volume 474, Issue 1, pp. 518-528, 1997.

[76]. R. Lionello, T. Torok, V. S. Titov, J. E. Leake, Z. Mikic, J. A. Linker, M. G. Linton, "The Contribution of Coronal Jets to the Solar Wind", The Astrophysical Journal Letters, Volume 831, Issue 1, article id. L2, 6 pp, 2016.

[77]. M. Opher, J. F. Drake, B. Zieger, T. I. Gombosi, "Magnetized jets driven by the Sun: the structure of the heliosphere revisited", arXiv: 1412.7687v2, 2014.

[78]. A. C. Sterling, K. L. Moore, D. A. Falconer, M. Adams, "Small scale filament eruptions as the driver of solar coronal hole jets", arXiv: 1705.03373v1, 2017.

[79]. P. F. Wyper, C. R. DeVore, S. K. Antiochos, “A breakout model for solar coronal jets with filaments”, arXiv: $1712.00134 v 2,2017$.

[80]. S.K. Antiochos, L.R. DeVore, J.A. Klimchuk, "A model for solar coronal mass ejection”, The Astrophysical Journal, 510, 485-493, 1999.

[81]. N. Gopalswamy, "History and development of coronal mass ejections as a key player in solar terrestrial relationship", Geoscience Letters, $3: 8,2016$

[82]. A Mishra and M Kumar “An insight into Space Weather”, Advanced Journal of Graduate Research, vol. 2, no. 1, pp. 46-57, 2017.

[83]. W.J. Wagner, "Coronal mass ejections", Annual Reviews in Astronomy and Astrophysics, 22, 267-289, 1984.

[84]. T.G. Forbes, “A review on the genesis of coronal mass ejections”, Journal of Geophysical Research, Vol. 105, No. A10, 23153-23165, 2000.

[85]. N. Gopalswamy, S. Akiyama, S. Yashiro, P. Makela, "Coronal mass ejection from sunspots and non-sunspots regions", arXiv: 0903.1087v1 [astro.ph-SR], 2009.

[86]. Nat Gopalswamy, "Coronal Mass Ejection and Space Weather", Climate and weather of the Sun and Earth System (CAWSES), Selected papers from the 2007 Kyoto symposium, Edited by T. Tsudu, R. Fujii, K. Shibata, M.A. Geller, pp 77-120, TERRAPUB, TOKYO, 2009.

[87]. B.C. Low, "Coronal mass ejections, magnetic flux ropes and solar magnetism", Journal of Geophysical Research, Vol. 106, No. A11, 25141-25163, 2001.

[88]. Emilia Kilpua, Hannu E.J. Koshkinen, Tuija I. Pulkkinen, "Coronal mass ejections and their sheath regions in the interplanetary space", Living Reviews in Solar Physics, 14:5, 2017.

[89]. W. VanTend, M. Kuperus, "The development of coronal electric current systems in active regions and their relation to filament and flares", Solar Physics, 59, 115-127, 1978.

[90]. T.G. Forbes, P.A. Isenberg, "A catastrophe mechanism for coronal mass ejection”, The Astrophysical Journal, 373, 294-307, 1991.

[91]. J.T. Karpen, S.K. Antiochos, C.R. DeVore, "The mechanism for the onset and explosive eruption of coronal mass ejection and eruptive flares”, The Astrophysical Journal, 760, 81(15pp), 2012.

[92]. N. Gopalswami, A. Lara, R.P. Lepping, M.L. Kaiser, D. Berdichevsky, O.C. St.Cyr, "Interplanetary acceleration of CMEs", Geophysical Research Letters, Vol. 27, No. 2, 145-148, 2000.

[93]. B. Vrsnak, N. Gopalswamy, "Influence of the aerodynamic drag on the motion of interplanetary ejecta", Journal of Geophysical Research, Vol. 107, No. A2, 1019, 2002.

[94]. B. Vrsnak, T. Zic, D. Vrbanec, M. Temmer, T. Rollett, C. Mostl, A. Veronica, J. Calogovic, M. Dumbovic, S. Lulic, Y.-J. Moon, A. Shanmugaraju, "propagation of interplanetary coronal mass ejections: The drag based model", Solar physics, 285, 295-315, 2013. 
Mishra et al., Adv. J. Grad. Res.; Vol. 6 Issue 1, pp: 1-13, July 2019

[95]. B. Schmeider, P. Demoulin, G. Aulanier, "Solar filament eruptions and their physical role in triggering coronal mass ejections", Advances in Space Research, Volume 51, Issue 11, 1967-1980, 2013.

[96]. Jiajia Liu, Yuming Wang, Chenglong Shen, Kai Liu, Zonghao Pan, S. Wong, "A solar coronal jet event triggers a coronal mass ejection”, The Astrophysical journal, 813, 115 (6pp), 2015.

[97]. P. Makela, N. Gopalswamy, S. Yashiro, S. Akiyama, H. Xie, E. Valtonen, "SEPs and CMEs during cycle 23”, Proceedings IAU Symposium No. 257, 2008.

[98]. $\quad$ Arnold O. Benz, "Flare Observations", Living Reviews in Solar Physics, 14:2, 2017.

[99]. T.G. Forbes, L.W. Acton, "Reconnection and field line shrinkage in a solar flare", The Astrophysical Journal, 459, 330-341, 1996.

[100]. Seiji Yashiro, Nat Gopalswamy, "Statistical relationship between solar flares and coronal mass ejections", Universal Heliophysical Processes, Proceedings IAU Symposium no. 257”, 2008.

[101]. J. Zhang, K.P. Dere, R.A. Howard, A. Vourlidas, “A study of the kinematic evolution of coronal mass ejection”, The Astrophysical Journal, 604, 420-432, 2004.

[102]. Huge Carmichael, "A process for flares", NASSP, 50, 451C, 1964.

[103]. T. Hirayama, "Theoretical model for flares and prominences I. Evaporating Flare Model", Solar Physics, 34, 323-338, 1974.

[104]. P. A. Sturrock, "Model of the high-energy phase of solar flare", Nature, 211, 695-697, 1996.

[105]. R.A. Kopp, G.W. Pneuman, "Magnetic reconnection in the corona and the loop prominence phenomenon", Solar Physics, 50, 85-98, 1976.

[106]. D. B. Seaton, A. E. Bartz, J. M. Darnel, "Observations of the formation, development, and structure of a current sheet in an eruptive solar flare", arXiv: 1610.06905v1, 2016.

[107]. N. Gopalswamy, S. Akiyama, S. Yashiro, "Major solar flares without coronal mass ejections", Proceedings IAU Symposium No. $257,2008$.

[108]. J. Heyvaerts, E.R. Priest, D.M. Rust, "An emerging flux model for solar flare phenomenon”, The Astrophysical Journal, 216, 123-137, 1977.

[109]. J.A. Miller, P.J. Cargill, G. Emslie, G.D. Holman, B.R. Dennis, T.N. LaRosa, R.M. Winglee, S.G. Benka, S. Tsuneta, "Critical issues for understanding particle acceleration in impulsive solar flares", Journal of Geophysical Research, Vol. 102, No. A7, Pages 14631-14659, 1997.

[110]. E.R. Priest, T.G. Forbes, "The magnetic nature of solar flares”, The Astronomy and Astrophysics Review, 10, 313-377, 2002.

\section{Publish your research article in AIJR journals- \\ $\checkmark$ Online Submission and Tracking \\ $\checkmark$ Peer-Reviewed \\ $\checkmark$ Rapid decision \\ $\checkmark$ Immediate Publication after acceptance \\ $\checkmark$ Articles freely available online \\ $\checkmark \quad$ Retain full copyright of your article. \\ Submit your article at journals.aijr.in}

\section{Publish your books with AIJR publisher-}

$\checkmark$ Publish with ISBN and DOI.

$\checkmark$ Publish Thesis/Dissertation as Monograph.

$\checkmark$ Publish Book Monograph.

$\checkmark$ Publish Edited Volume/ Book.

$\checkmark$ Publish Conference Proceedings

$\checkmark$ Retain full copyright of your books.

Submit your manuscript at books.aijr.org 